\title{
EFFECT OF SURFACE PRE-TREATMENTS ON BONDING PERFORMANCE OF ZIRCONIA CERAMIC WITH RESIN CEMENTS
}

\author{
Walid Al-Zordk* , Heba Shoukry** and Amal Sakrana***
}

\begin{abstract}
Purpose: To establish an effective bonding for zirconia, the aim of this study was to evaluate the influence of air-borne particle abrasion, Piranha acid etching and hot acid etching pre-treatments on bond strength of zirconia with self-adhesive resin cements (Panavia SA, TheraCem) and conventional adhesive resin cement (Panavia F2.0). Also, the effect of Silano-Pen treatment on the bond strength of zirconia to resin cements was evaluated.
\end{abstract}

Materials and Methods: Eighteen zirconia blocks were cut, sintered and divided into three groups ( $n=6)$ : air-borne particle abrasion, Piranha acid etching $\left(3 \mathrm{H}_{2} \mathrm{SO}_{4}: 1 \mathrm{H}_{2} \mathrm{O}_{2}\right)$, and hot acid etching $\left(1 \mathrm{HNO}_{3}: 1 \mathrm{HF}\right)$. From each group, the bonding surfaces of three zirconia blocks were treated with Silano-Pen. Each zirconia block was bonded to its corresponding composite block utilizing either Panavia SA, TheraCem or Panavia F2.0. Each ceramic/ resin / composite assembly was sectioned perpendicular to the bonding interface to obtain microbars of $1 \mathrm{~mm}^{2}$ thickness. A total of 180 microbars were subjected to 10000 thermal cycles between $5^{\circ} \mathrm{C}$ and $55^{\circ} \mathrm{C}$ with dowel time of 30 seconds. Each microbar was subjected to tensile force until de-bonding. The data was statistically analyzed.

Results: The hot acid showed the highest $\mu$ TBS $(21.96 \pm 5.86 \mathrm{MPa})$ followed by air-borne particle abrasion $(16.40 \pm 6.23 \mathrm{MPa})$ and the lowest was Piranha $(15.04 \pm 7.12 \mathrm{MPa})$. With Panavia SA, there was significant difference $(p=.035)$ between $\mu$ TBS with Silano-Pen in air-borne particle abrasion and Piranha groups, also there was significant difference $(p=.004)$ between Piranha and hot acid groups. With TheraCem, there was significant difference $(p=.008)$ between $\mu$ TBS with Silano-Pen in air-borne particle abrasion and Piranha groups, also there was significant difference $(p=.003)$ between air-borne particle abrasion and hot acid groups. The interaction between cement and Silano-Pen was insignificant $(p=.067)$.

Conclusions: Pre-treatment method and type of adhesive resin cement influences the effectiveness of bonding of zirconia. The hot acid etching recorded the highest bond strength, whereas the lowest was recorded with Piranha etching. Silano-Pen treatment after hot acid etching improved the bonding of zirconia to adhesive resin cement. The self-adhesive MDP-containing resin cement (Panavia SA) enhanced the effectiveness of the bond strength with zirconia.

KEY WORDS: Zirconia, bonding, self-adhesive, hot acid, Piranha, sandblasting.

\footnotetext{
* Assistant Professor, Department of Fixed Prosthodontics, Mansoura University, Mansoura, Dkahlia, Egypt. ** Postgraduate Student, Department of Fixed Prosthodontics, Mansoura University, Mansoura, Dkahlia, Egypt. *** Professor, Department of Fixed Prosthodontics, Mansoura University, Mansoura, Dkahlia, Egypt.
} 


\section{INTRODUCTION}

One of the limitations for the clinical durability of zirconia is its limited ability to bond with resin cement because zirconia is a glass-free polycrystalline microstructure, non-etchable and chemically inert with low surface energy. ${ }^{1-3}$ Various surface treatment methods were employed, to enhance bonding with zirconia, such as air-borne particle abrasion, hot chemical etching, and pyrochemical silica coating. Air-borne particle abrasion increases the surface wettability, surface roughness and provides micromechanical undercuts. ${ }^{4}$ However, air-borne particle abrasion results in structural defects and induction of sharp cracks that enhance radial cracking during function. ${ }^{5}$ Piranha etching solution is a combination of hydrogen peroxide and sulfuric acid which a strong oxidizing corrosive agent used to remove the organic impurities and hydroxylate surfaces. ${ }^{1,6}$ Hot chemical etching improves the surface roughness through a corrosion controlled process which is based on removing the less well arranged and high energy peripheral atoms resulting in wider grain boundaries. ${ }^{7,8}$ The using of hydrofluoric acid in different temperature and concentrations could produce modifications in zirconia surface. ${ }^{9}$ Moradabadi et $\mathrm{a}^{10}$ studied the effect of micromechanical and chemical surface treatments on bond strength of zirconia to resin cement and concluded that the air-borne particle abrasion treatment prior to acid etching process enhanced the shear bond strength. Pyrochemical silica coating depends on formation of a silica layer through the chemical reactions of silane at high temperature. ${ }^{4,11}$

Contemporary resin cements were classified into self-adhesive resin cements and conventional resin cements. ${ }^{12}$ Self-adhesive resin cement contains acid functionalized monomers and conventional methacrylate monomers. Based on the functional acidic monomers, there are two popular groups; methacrylate monomers with carboxylic acid groups such as 4-META based cements and PMGDM based cements, or with phosphoric acid groups such as MDP based cements and BMP based cements. ${ }^{13}$
Oyaguie et $a{ }^{14}$ evaluated the hydrolytic stability of different resin cements when bonded to zirconia and concluded that water aging played an important role in the degradation of the bond strength and the bond durability depended mainly on the cement selection rather than the applied surface treatment.

To study the adhesion of the resin cement to zirconia, there are various testing methods such as macro-shear, microshear, macrotensile and microtensile tests. It is critical that the bonding interface must be the most stressed zone, regardless of the applied test methodology. With micro-tensile test, the small interfacial bonding zone $\left(1 \mathrm{~mm}^{2}\right)$ and small dimension shows more homogeneous stress distribution; therefore, it presents more sensitive evaluation of bond performance when specimens are aligned correctly. ${ }^{15,16}$

The main limitation of zirconia based restoration is its low adhesive potential and the conventional adhesive techniques do not produce a high enough bond strength. ${ }^{17,18}$ To establish a reproducible, effective and applicable bonding protocol for zirconia restorations, the objective of the present study was to evaluate the influence of air-borne particle abrasion, Piranha acid etching and hot acid etching pre-treatments on bond strength of zirconia with self-adhesive resin cements (Panavia SA Cement Plus and TheraCem) and conventional adhesive resin cement (Panavia F2.0). Also, the effect of Silano-Pen treatment on the bond strength of zirconia to self-adhesive resin cements and conventional resin cements. Thus, the null hypotheses of this study were there no differences in the effect of surface pre-treatment methods on bond strength of zirconia with adhesive resin cements.

\section{MATERIALS AND METHODS}

Partially sintered zirconia blocks (inCoris TZI C, Sirona Dental, Germany) were cut using precision cutting machine (Isomet 4000, Buehler Ltd, Lake Bluff, IL, USA) to obtain eighteen zirconia blocks measuring $10 \mathrm{~mm}$ length, $10 \mathrm{~mm}$ width and 6 
mm thickness. All zirconia blocks were sintered in zirconia sintering furnace (Sirona inFire HTC, Sirona Dental Systems, GmbH, Germany) according to manufacturer's instructions. After sintering, the dimension of each zirconia block (8 mm length, 8 $\mathrm{mm}$ width and $4.8 \mathrm{~mm}$ thickness) was measured using digital caliper (Mitutoyo, Tokyo, Japan) to verify the volumetric shrinkage after sintering. After finishing using a zirconia-specific finishing kit (Eve Ernst Vetter GmbH, Germany), all zirconia blocks were ultrasonically cleaned in distilled water for 10 minutes, then air dried..$^{19}$ A light-cure composite (Tetric N-Ceram, Ivoclar Vivadent) was used to fabricate eighteen composite blocks using Teflon mold $(8 \mathrm{~mm}$ length, $8 \mathrm{~mm}$ width and 4.8 $\mathrm{mm}$ thickness). After curing, the bonding surface of each composite block was polished, ultrasonically cleaned and air dried. Eighteen zirconia blocks were equally divided according to the surface treatment into three main groups $(n=6)$ : air-borne particle abrasion group, Piranha group (air-borne particle abrasion and Piranha acid etching) and hot acid group (air-borne particle abrasion and hot acid etching). Each group was divided into two subgroups $(n=3)$ either directly bonded with no further treatment or treated with Silano-Pen before bonding of zirconia with resin cements. Panavia SA Cement Plus Cement Plus self-adhesive resin cement (Kuraray Noritake Dental, Tokyo, Japan), TheraCem self-adhesive resin cement (BISCO Inc, USA) and Panavia F2.0 adhesive resin cement (Kuraray Noritake Dental, Tokyo, Japan) were the resin cements used for bonding.

For air-borne particle abrasion group, the bonding surfaces of zirconia blocks were particle abraded with $50 \mu \mathrm{m} \mathrm{Al}_{2} \mathrm{O}_{3}$ at a distance of $10 \mathrm{~mm}$ and perpendicular to the surface. ${ }^{20}$ For Piranha group, the bonding surfaces of zirconia blocks were particle abraded as in air-borne particle abrasion group. Then, the zirconia blocks were immersed in a glass beaker containing Piranha acid solution $\left(3 \mathrm{H}_{2} \mathrm{SO}_{4}: 1 \mathrm{H}_{2} \mathrm{O}_{2}\right)$ for 4 days. ${ }^{1}$ The acid solution was daily replaced by fresh solution. The Piranha solution was prepared from a mixture of $96 \%$ sulfuric acid (Al Nasr Pharmaceutical Chemicals Co., Egypt) and 30\% hydrogen peroxide (Piochem Co., Egypt). As regard hot acid group, the bonding surfaces were air abraded and immersed in polyethylene beaker containing the hot chemical etching solution $\left(1 \mathrm{HNO}_{3}: 1 \mathrm{HF}\right)$ which was heated up to $100{ }^{\circ} \mathrm{C}$ in water bath for 25 minutes. ${ }^{21}$ The hot chemical etching solution was prepared as a mixture of $69 \%$ nitric acid (Honeywell International Inc., Burdick and Jackson, Seelze, Germany) and 48\% hydrofluoric acid (Honeywell International Inc., Riedel-de Haën, Seelze, Germany). Finally, all zirconia blocks were rinsed with distilled water, ultrasonically cleaned in distilled water for 10 minutes and air dried. For each group, a representative zirconia block was pre-treated for SEM evaluation of the surface pretreatments. From each group, the bonding surface of three zirconia block was subjected to Silano-Pen treatment. The bonding surfaces of nine zirconia blocks were heated with the pale blue reactive flame zone of Silano-Pen device (Bredent $\mathrm{GmbH}$, Senden, Germany) according to the manufacturer's instructions. After the surface was cooled down to room temperature, the silane liquid (Silane Haftvermittler, Bredent, Senden, Germany) was applied using a disposable brush and left for 30 seconds.

Each zirconia block was bonded to its corresponding composite block utilizing either Panavia SA Cement Plus, TheraCem or Panavia F2.0 resin cements according to manufacturer's recommendations. The bonding procedures were performed under a static load of $1 \mathrm{~kg}$ to ensure a uniform cement layer. ${ }^{22}$ The light curing was performed from all directions for each cement according to the recommendations of the manufacturers. The ceramic/resin /composite assemblies were stored in distilled water at $37^{\circ} \mathrm{C}$ for 24 hours. $^{23}$ Using a cutting machine (Isomet 4000), each assembly was sectioned perpendicular 
to the bonding interface area to obtain microbars of $1 \mathrm{~mm}^{2}$ thickness. For each microbar, the crosssectional area of the bond interface was verified using a digital caliper (Mitutoyo, Tokyo, Japan). Microbars were examined under stereomicroscope (MA 100 Nikon, Japan) at 50x magnification for selection of the intact specimens that are free from any microcracks.

A total of 180 microbars were assigned for testing of the bond strength as presented in figure 1. All specimens were subjected to 10000 thermal cycles (SD Mechatronics Thermocycler, Westerham, Germany) between $5{ }^{\circ} \mathrm{C}$ and $55^{\circ} \mathrm{C}$ with a dowel time of 30 seconds. ${ }^{24}$ Each microbar was subjected to tensile force through a universal testing machine (3345, Instron, 2519-104, 3345, Canton, MA, USA) until de-bonding. The mean value of the bond strength for each specimen was calculated in Mega Pascale (MPa) using the machine software (Bluehill Lite software, Instron, MA, USA) through dividing the load at failure $(\mathrm{N})$ by the adhesive area $\left(\mathrm{mm}^{2}\right)$. Statistical analysis was done using statistical software (SPSS Statistics for Windows version 22).

\section{RESULTS}

Means and standard deviations of microtensile bond strength values are presented in Table 1. Normal and relative (marginal) distributions of data was tested by using Shapiro-Wilk's test and Levene's test that revealed normal data distribution. Regardless the bonding procedure within each pretreatment, one-way ANOVA showed significant difference $(\mathrm{F}=6.810, p=.000)$ between air-borne particle abrasion, Piranha acid etching and hot acid etching surface pre-treatments. Post Hoc multiple comparisons revealed significant difference between the main surface pre-treatments with the highest bond strength in hot acid group and the lowest bond strength in Piranha group (Table 2). As regard to resin cement, there was significant difference between Panavia SA and TheraCem, and between Panavia SA and Panavia F2.0 (Table 3).
TABLE (1) Mean and standard deviation of $\mu$ TBS values of the test groups.

\begin{tabular}{|c|c|c|c|c|c|}
\hline & & & $\mathrm{N}$ & Mean & $\begin{array}{c}\text { Stand } \\
\text { deviation }\end{array}$ \\
\hline \multirow{7}{*}{ 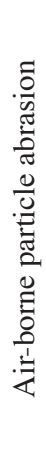 } & \multirow{3}{*}{ 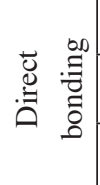 } & Panavia SA & 10 & 21.516 & 7.33 \\
\hline & & TheraCem & 10 & 15.350 & 1.84 \\
\hline & & Panavia F2.0 & 10 & 20.649 & 2.52 \\
\hline & \multirow{3}{*}{ 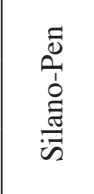 } & Panavia SA & 10 & 20.185 & 4.17 \\
\hline & & TheraCem & 10 & 10.640 & 4.08 \\
\hline & & Panavia F2.0 & 10 & 10.068 & 4.25 \\
\hline & & Total & 60 & 16.401 & 6.23 \\
\hline \multirow{7}{*}{ 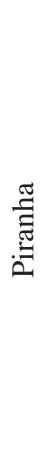 } & \multirow{3}{*}{ 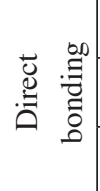 } & Panavia SA & 10 & 23.077 & 5.11 \\
\hline & & TheraCem & 10 & 11.406 & 3.77 \\
\hline & & Panavia F2.0 & 10 & 5.699 & 0.80 \\
\hline & \multirow{3}{*}{ 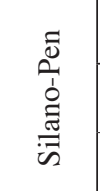 } & Panavia SA & 10 & 12.982 & 4.31 \\
\hline & & TheraCem & 10 & 19.731 & 7.11 \\
\hline & & Panavia F2.0 & 10 & 17.352 & 3.43 \\
\hline & & Total & 60 & 15.041 & 7.12 \\
\hline \multirow{7}{*}{ 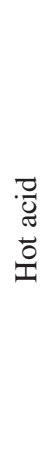 } & \multirow{3}{*}{ 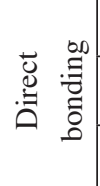 } & Panavia SA & 10 & 25.120 & 4.55 \\
\hline & & TheraCem & 10 & 17.494 & 8.78 \\
\hline & & Panavia F2.0 & 10 & 22.181 & 6.91 \\
\hline & \multirow{3}{*}{ 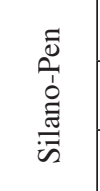 } & Panavia SA & 10 & 23.001 & 3.04 \\
\hline & & TheraCem & 10 & 20.805 & 5.54 \\
\hline & & Panavia F2.0 & 10 & 23.199 & 4.59 \\
\hline & & Total & 60 & 21.967 & 5.86 \\
\hline
\end{tabular}

With Panavia SA, there was significant difference ( $p=.035$ ) between bond strength with SilanoPen in air-borne particle abrasion and Piranha, also there was significant difference $(p=.004)$ between Piranha and hot acid. However, there was no significant difference $(p=.408)$ between bond strength in air-borne particle abrasion and hot acid with SilanoPen application (table 4). Regarding TheraCem, there was significant difference $(p=.008)$ between bond strength with Silano-Pen in air-borne particle 
abrasion and Piranha, also there was significant difference $(p=.003)$ between air-borne particle abrasion and hot acid. However, there was no significant difference $(p=.752)$ between bond strength in Piranha and hot acid with Silano-Pen application. With Panavia F2.0, there was significant difference with direct bonding and with Silano-Pen $(p=.000$ and .034 respectively) between bond strength in airborne particle abrasion and Piranha.
For interaction between tested variables, the surface pre-treatment and cement significantly affect the bond strength values. Also, the interaction between the surface pre-treatment and cement, and surface pre-treatment and SilanoPen was significant (Table 5). SEM evaluation of each surface pre-treatments are presented in Figures 1-4.

TABLE (2) Post Hoc multiple comparisons using LSD of the three main surface pre-treatments.

\begin{tabular}{|c|c|c|c|c|c|c|}
\hline \multicolumn{2}{|c|}{ Main surface treatments } & \multirow{2}{*}{$\begin{array}{c}\text { Mean Difference } \\
(\mathrm{I}-\mathrm{J})\end{array}$} & \multirow{2}{*}{ Std. Error } & \multirow{2}{*}{ Sig. } & \multicolumn{2}{|c|}{ 95\% Confidence Interval } \\
\hline (I) & (J) & & & & Lower Bound & Upper Bound \\
\hline \multirow{2}{*}{$\begin{array}{c}\text { Air-borne } \\
\text { particle } \\
\text { abrasion }\end{array}$} & Piranha & $2.7542 *$ & .97954 & .006 & .8181 & 4.6903 \\
\hline & Hot acid & $-4.3410 *$ & .97954 & .000 & -6.2771 & -2.4048 \\
\hline \multirow{2}{*}{ Piranha } & Air-borne particle abrasion & $-2.7542 *$ & .97954 & .006 & -4.6903 & -.8181 \\
\hline & Hot acid & $-7.0952 *$ & .97954 & .000 & -9.0313 & -5.1590 \\
\hline \multirow{2}{*}{ Hot acid } & Air-borne particle abrasion & $4.3410 *$ & .97954 & .000 & 2.4048 & 6.2771 \\
\hline & Piranha & $7.0952 *$ & .97954 & .000 & 5.1590 & 9.0313 \\
\hline
\end{tabular}

TABLE (3) Post Hoc multiple comparisons using LSD of Panavia SA Cement Plus, TheraCem and Panavia F2.0 adhesive resin cements.

\begin{tabular}{|c|c|c|c|c|c|c|}
\hline \multicolumn{2}{|c|}{ Resin Cement } & \multirow{2}{*}{$\begin{array}{c}\text { Mean Difference } \\
(\mathrm{I}-\mathrm{J})\end{array}$} & \multirow{2}{*}{ Std. Error } & \multirow{2}{*}{ Sig. } & \multicolumn{2}{|c|}{ 95\% Confidence Interval } \\
\hline (I) & $(\mathrm{J})$ & & & & Lower Bound & Upper Bound \\
\hline \multirow{2}{*}{ Panavia SA } & TheraCem & $5.5791 *$ & .97954 & .000 & 3.6430 & 7.5152 \\
\hline & Panavia F2.0 & $4.1732 *$ & .97954 & .000 & 2.2371 & 6.1093 \\
\hline \multirow{2}{*}{ TheraCem } & Panavia SA & $-5.5791 *$ & .97954 & .000 & -7.5152 & -3.6430 \\
\hline & Panavia F2.0 & -1.4059 & .97954 & .153 & -3.6430 & .5302 \\
\hline \multirow{2}{*}{ Panavia F2.0 } & Panavia SA & $-4.1732 *$ & .97954 & .000 & -6.10 .93 & -2.2371 \\
\hline & TheraCem & 1.4059 & .97954 & .153 & -.5302 & 3.3420 \\
\hline
\end{tabular}


TABLE (4) Comparison of $\mu$ TBS values with Panavia SA Cement Plus, TheraCem and Panavia F2.0 among groups.

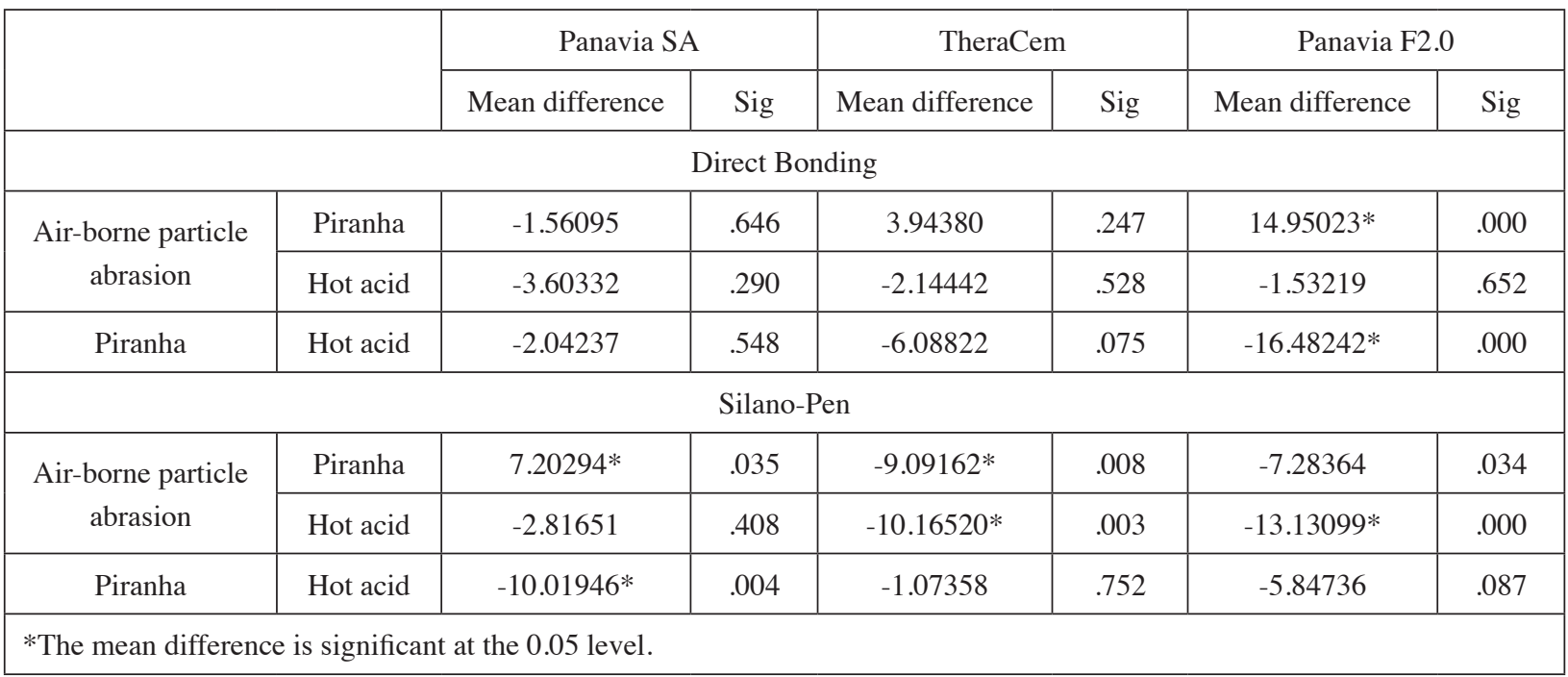

TABLE (5) The interaction between the study variables.

\begin{tabular}{|c|c|c|c|c|c|}
\hline Source of variation & Type II Sum of Squares & Df & Mean Square & F value & $\mathrm{p}$ value \\
\hline Main surface treatment (A) & 1535.418 & 2 & 767.709 & 26.671 & .000 \\
\hline Silano-Pen treatment (B) & 1.819 & 1 & 1.819 & .063 & .802 \\
\hline Cement (C) & 1010.371 & 2 & 505.186 & 17.550 & .000 \\
\hline$(\mathrm{A}) *(\mathrm{~B})$ & 256.909 & 2 & 128.454 & 4.463 & .013 \\
\hline$(\mathrm{A}) *(\mathrm{C})$ & 788.371 & 4 & 197.093 & 6.847 & .000 \\
\hline$(\mathrm{B}) *(\mathrm{C})$ & 158.229 & 2 & 79.115 & 2.748 & .067 \\
\hline$(\mathrm{A}) *(\mathrm{~B}) *(\mathrm{C})$ & 697.073 & 4 & 174.268 & 6.054 & .000 \\
\hline Error & 4145.015 & 144 & 28.785 & & \\
\hline Total & 89412.664 & 180 & & & \\
\hline
\end{tabular}

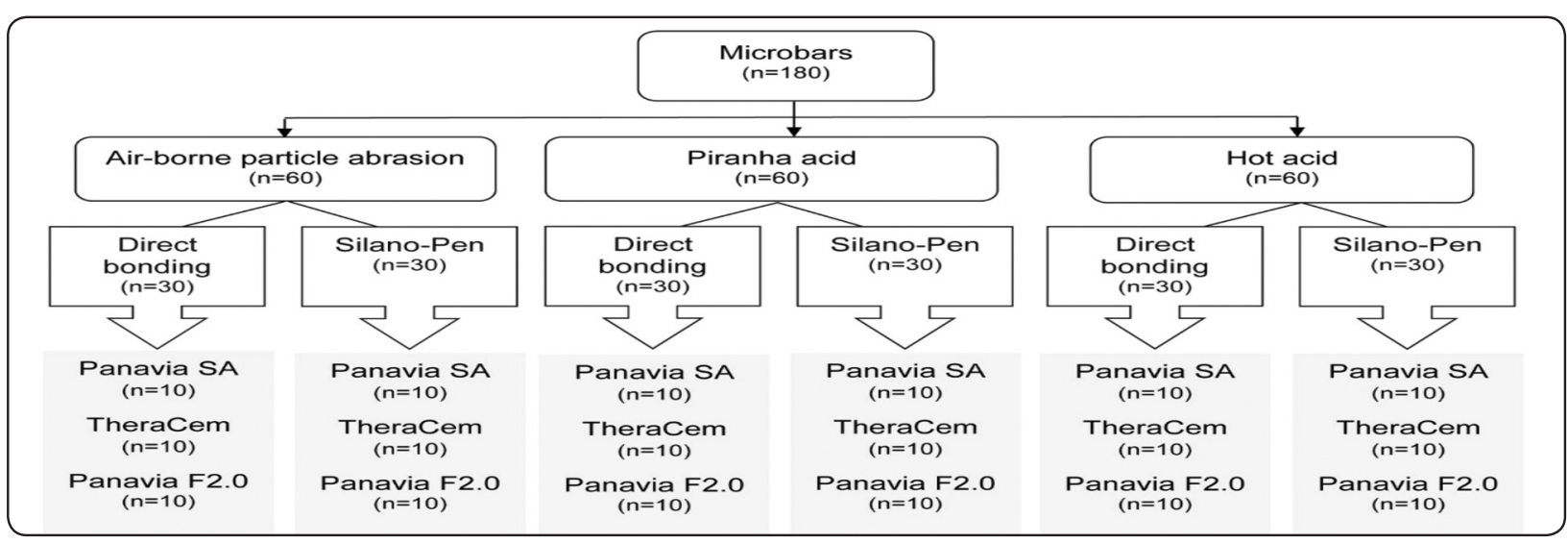

Fig. (1) Follow chart detailing the study setup. 


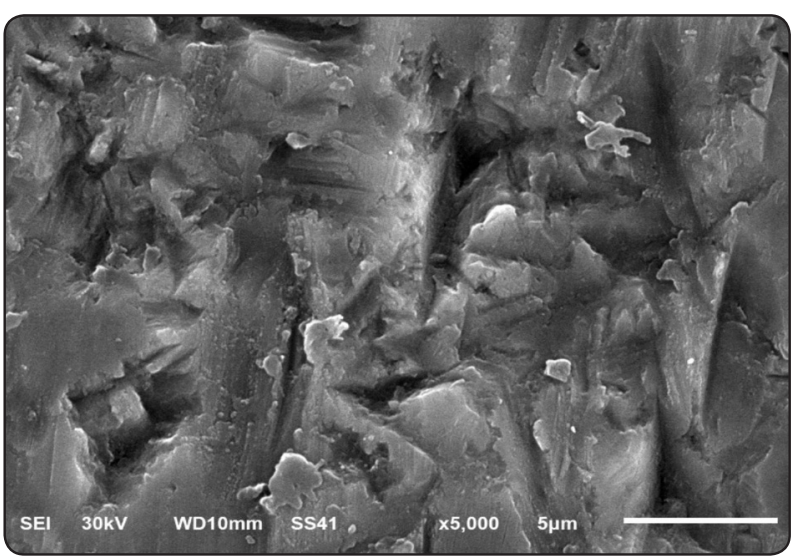

Fig. (2) SEM micrograph (5000x) showing the air abraded surface of zirconia.

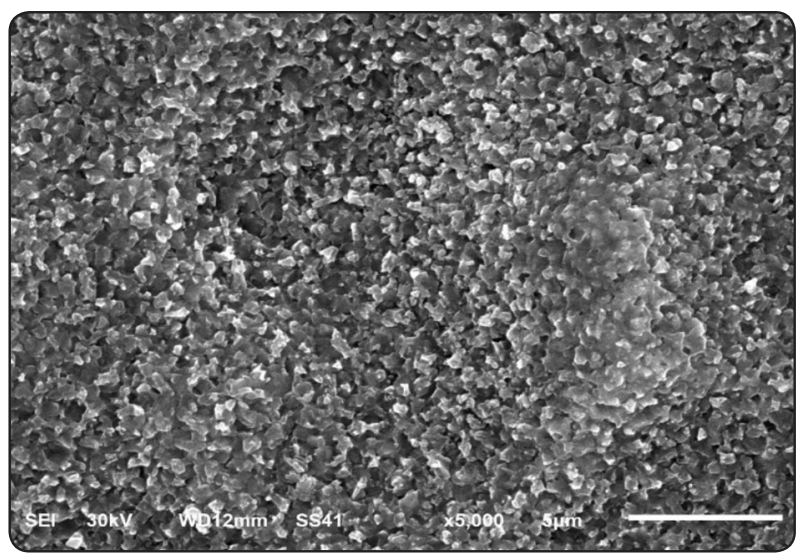

Fig. (4) SEM micrograph (5000x) showing the zirconia surface after etching with hot acid solution.

\section{DISCUSSION}

There are several methods for achieving a high bond strength with zirconia, but this bond should be able to withstand the surrounding oral environment over years. ${ }^{25}$ Recently, adhesive strategies that combine mechanical and chemical pre-treatment have been developed to improve the bond durability between resin cement and zirconia. ${ }^{26}$ Air-borne particle abrasion with aluminum oxide particles with a particle size of $50 \mu \mathrm{m}$ is a common and simple procedure to produce micro-mechanical roughness. ${ }^{3,27}$ Also, the usage of $50 \mu \mathrm{m}$ aluminum oxide particles has a less harmful effect of the surface topography of zirconia when compared with

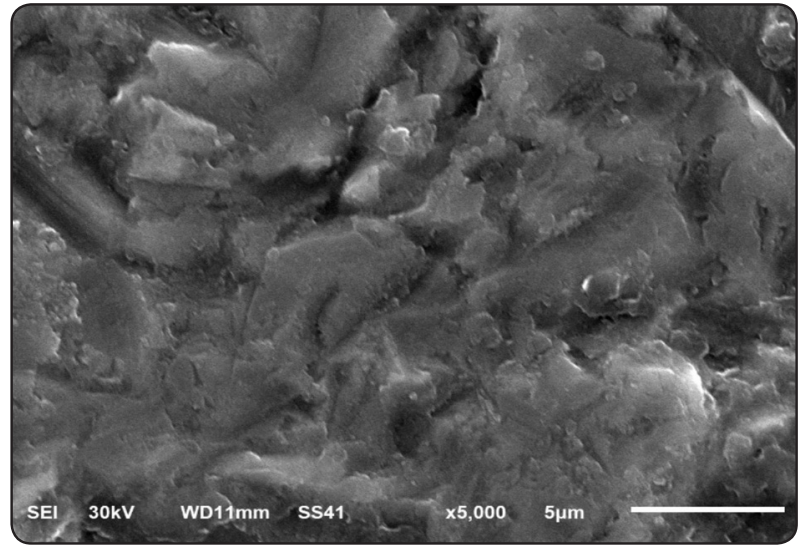

Fig. (3) SEM micrograph (5000x) showing the zirconia surface after etching with Piranha acid solution.

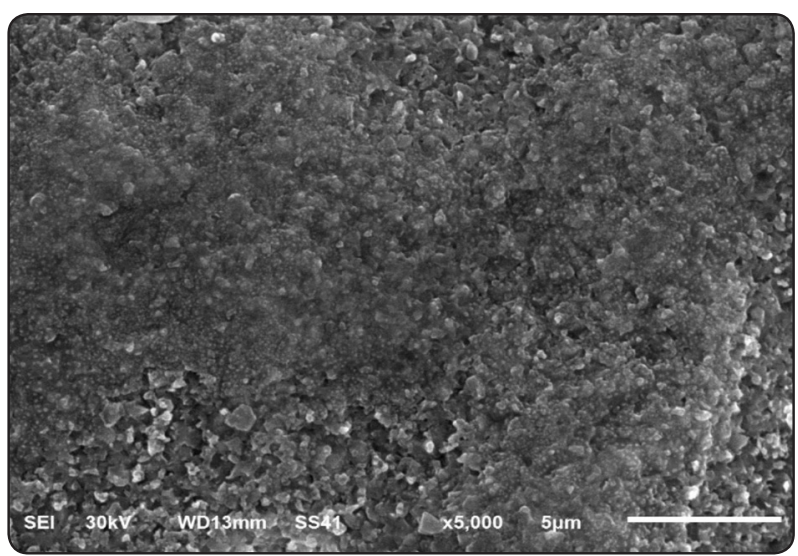

Fig. (5) SEM micrograph (5000x) showing the zirconia surface after etching with hot acid solution and Silano-Pen treatment.

$120 \mu \mathrm{m}$ aluminum oxide particles. ${ }^{28}$ In the present study, Piranha acid etching solution was prepared according to Lohbauer et al $^{1}$ who observed that $96 \%$ sulfuric acid and $30 \%$ hydrogen peroxide with ratio of 3:1 for four days exhibited effective chemical preconditioning with effective hydroxylation of abrade zirconia surface. Also, hot acid etching solution was prepared according to Liu et $\mathrm{al}^{21}$ who found that $69 \%$ nitric acid and $48 \%$ hydrofluoric acid with ratio of $1: 1$ at $100^{\circ} \mathrm{C}$ for 25 minutes resulted in improving the dissolution rate of zirconia grains with increased roughness. In the present study, airborne particle abrasion was applied before hot acid etching to get the advantages of both methods. 
The hot acid etching pre-treatment showed the highest micro-tensile bond strength values $(21.96 \pm 5.86 \mathrm{MPa})$ followed by air-borne particle abrasion (16.40 $\pm 6.23 \mathrm{MPa})$. The Piranha acid etching resulted in the lowest micro-tensile bond strength value $(15.04 \pm 7.12 \mathrm{MPa})$. The rank of micro-tensile bond strength from lowest was Piranha "air-borne particle abrasion" hot acid. Therefore, the first part of null hypothesis was rejected. The results of the present study were coincided with that of Casucci et $\mathrm{al}^{22}$ who found that zirconia treated with hot acid solution recorded higher bond strength than those treated with air-borne particle abrasion. Another study showed higher bond strength with hot acid etching than air-borne particle abrasion. ${ }^{29}$ The hot acid etching improves the surface roughness and remove the superficial ceramic layer resulting in a homogenous granular and porous texture of zirconia. ${ }^{8}$ Studies showed that the bond strength and durability after Piranha acid etching was lower than that after air-borne particle abrasion. ${ }^{6,30}$ The inferior bond strength and durability is related to the unstable bond between the resin cement and the hydroxyl groups produced by Piranha solution. ${ }^{6}$ Additionally, surface conditioning with Piranha solution clean and hydroxylates the surface without formation of undercuts which are particularly important in micromechanical interlocking with the resin cement. ${ }^{30}$

In contrary, Moradabadi et $\mathrm{al}^{10}$ showed that the zirconia treated with air-borne particle abrasion recorded higher bond strength than that treated with air-borne particle abrasion and $\mathrm{HF} / \mathrm{HNO}_{3}$ etching at room temperature for two minutes. The addition of this etching solution to the abraded zirconia surface leading to deformation of surface roughness created by air-borne particle abrasion leading to deformation of this roughness to be rounded and which results in reduction of the micromechanical retention. ${ }^{10}$ In the current study, the acid solution was performed for 25 minutes at $100^{\circ} \mathrm{C}$. That temperature with hot acid etching has an essential role in molecular motion as the higher the temperature, the protons become more easily ionized leading to more acidic effect. ${ }^{8}$ Another study reported that the addition of Piranha solution to the abraded zirconia had higher bond strength than air-borne particle abrasion using $110 \mu \mathrm{m}$ particle size. ${ }^{1}$ The possible explanation is that the aggressive air abrasion using bigger particle size could result in ditching between resin cement and zirconia surface. ${ }^{31}$

Concerning Silano-Pen, the results showed that the interaction between main surface pre-treatment and Silano-Pen was significant, while no significant interaction between Silano-Pen and cement. The heat treatment using Silano-Pen could improve the surface hydroxylation resulting in a more reactive zirconia surface..$^{31}$ Moreover, Silano-Pen enhances the surface wettability and produces a dense scattering nanosilica grains leading to a stratified surface topography. ${ }^{32,33}$ Additionally, the role of air-borne particle abrasion with hot acid etching in creation and improving the surface roughness could not be neglected. ${ }^{20}$

In the present study, a comparison was held between MDP-containing conventional resin cement (Panavia F2.0) which is commonly used in researches and two self-adhesive resin cements which are methacrylate-based (TheraCem) and MDP-containing (Panavia SA) self-adhesive resin cements. The results of the present study showed that Panavia SA Cement Plus showed high bond strength. These results could be related to the high content of acidic phosphate functional monomers in self-adhesive resin cement which could increase hydrophilicity of the cement resulting in hydrolytic degradation due to the higher water sorption. ${ }^{34}$ Tanis et $\mathrm{al}^{35}$ showed that the using of MDP-containing resin cement improved the bond strength with airborne particle abrasion. Another study reported that the abraded zirconia specimens bonded with methacrylate-based cement showed lower bond strength than bonded with MDP-containing resin cement as the MDP monomers creates a chemical interaction with zirconia. ${ }^{36}$ Also, the results of the present study revealed that the highest bond strength 
with hot acid etching. The hot acid etching improves the surface roughness by dissolving the less well arranged peripheral atoms of zirconia surface resulting in larger grain boundaries formation which increase mechanical interlocking with resin cement with no phase transformation. ${ }^{23}$

With Panavia F2.0, there was significant decrease in the bond strength $(5.69 \pm 0.80 \mathrm{MPa})$ after aging with Piranha group. This bond strength value is considered a very low value for acceptable clinical bonding as the range of 10-13 MPa was suggested as the minimum clinically acceptable bond strength. ${ }^{37,38}$ Although the increased inorganic filler $(59 \mathrm{vol} \%)$ has a significant role in improving wear resistance, mechanical properties and reducing polymerization shrinkage, it affect the proper viscosity and the suitable film thickness. ${ }^{39}$ Moreover, this cement needs hand mixing which possibly leading to incorporation of air bubbles and resulting in reduction of the mechanical properties. ${ }^{40}$

New zirconia ceramics for dental restorations are continually under development, only one type of zirconia was tested in the present study. The results obtained should be verified in future studies in comparison with more surface condition methods and with more prolonged aging. The specimens were produced and examined under ideal conditions which may not reflect actual clinical conditions. Further clinical studies are needed to confirm the relationship between surface pre-treatment, SilanPen and cement to confirm the durability of the bonding protocol for zirconia restorations.

\section{CONCLUSIONS}

Within the limitations of the current study, it was concluded that:

1. The surface pre-treatment method and type of adhesive resin cement influences the effectiveness of bonding with zirconia-based restoration.

2. The hot acid etching pre-treatment recorded the highest bond strength, whereas the lowest bond strength was recorded with Piranha acid etching pre-treatment.
3. Silano-Pen treatment after hot acid etching improved the bonding of zirconia to adhesive resin cement more than after air-borne particle abrasion.

4. Among the tested adhesive resin cements, the self-adhesive MDP-containing resin cement (Panavia SA Cement Plus) enhanced the effectiveness of the bond strength with zirconia restoration.

\section{REFERENCES}

1. Lohbauer U, Zipperle M, Rischka K, Petschelt A, Müller FA. Hydroxylation of dental zirconia surfaces: characterization and bonding potential. J Biomed Mater Res B Appl Biomater 2008; 87: 461-467.

2. He M, Zhang Z, Zheng D, Ding N, Liu Y. Effect of sandblasting on surface roughness of zirconia-based ceramics and shear bond strength of veneering porcelain. Dent Mater J 2014; 33: 778-785.

3. Tzanakakis E G, Tzoutzas IG, Koidis PT. Is there a potential for durable adhesion to zirconia restorations? A systematic review. J Prosthet Dent 2016; 115: 9-19.

4. Matinlinna JP, Lung CYK, Tsoi JKH .Silane adhesion mechanism in dental applications and surface treatments: A review. Dent Mater 2018; 34: 13-28.

5. Wang G, Zhang S, Bian C, Kong H. Effect of zirconia surface treatment on zirconia/veneer interfacial toughness evaluated by fracture mechanics method. J Dent 2014; 42: 808-815.

6. Hallmann L, Ulmer P, Lehmann F, Wille S, Polonskyi O, Johannes M, et al. Effect of surface modifications on the bond strength of zirconia ceramic with resin cement resin. Dent Mater 2016; 32: 631-639.

7. Lv P, Yang $x$, Jiang T. Influence of hot-etching surface treatment on zirconiaresin shear bond strength. Materials 2015; 30: 8087-8096.

8. Xie H, Chen C, Dai W, Chen G, Zhang F. In vitro shortterm bonding performance of zirconia treated with hot acid etching and primer conditioning etching and primer condioning. Dent Mater J 2013; 32: 928-938.

9. Cho JH, Kim SJ, Shim JS, Lee KW. Effect of zirconia surface treatment using nitric acid-hydrofluoric acid on the shear bond strengths of resin cements. J Adv Prosthodon 2017; 9: 77-84. 
10. Moradabadi A, Roudsari SES, Yekta BE, Rahbar N. Effects of surface treatment on bond strength between dental resin agent and zirconia ceramic. Mater Sci Eng C Mater Biol Appl 2014; 34: 311-317.

11. Radovic I, Monticelli F, Goracci C, Vulicevic ZR, Ferrari M. Self-adhesive resin cements: a literature review. J Adhes Dent. 2008; 10: 251-258.

12. Ferracane JL, Stansbury JW, Burke FJ. Self-adhesive resin cements - chemistry, properties and clinical considerations. J Oral Rehabil 2011; 38: 295-314.

13. Manso AP, Carvalho RM. Dental cements for luting and bonding restorations: self-adhesive resin cements. Dent Clin North Am 2017; 61: 821-834.

14. Oyagüe RC, Monticelli F, Toledano M, Osorio E, Ferrari M, Osorio R. Effect of water aging on microtensile bond strength of dual-cured resin cements to pre-treated sintered zirconium-oxide ceramics. Dent Mater 2009; 25: 392-399.

15. Pashley DH, Carvalho RM, Sano H, Nakajima M, Yoshiyama M, Shono Y, et al. The microtensile bond test: a review. J Adhes Dent 1999; 1: 299-309.

16. Smith RL, Villanueva C, Rothrock JK, Garcia-Godoy CE, Stoner BR, Piascik JR, et al. Long-term microtensile bond strength of surface modified zirconia. Dent Mater. 2011; 27: 779-785

17. Negreiros WM, Ambrosano GMB, Giannini M. Effect of cleaning agent, primer application and their combination on the bond strength of a resin cement to two yttrium-tetragonal zirconia polycrystal zirconia ceramics. Eur J Dent 2017;11: 6-11.

18. Li RWK, Chow TW, Matinlinna JP. Ceramic dental biomaterials and CAD/CAM technology: state of the art. J Prosthodont Res 2014; 58: 208-216.

19. Larabi H, Cetik S, Thai Ha H, Atash R, Ha HT. In vitro study of bonding strength of zirconia on dentin using different adhesive systems. Int J Prosthodon. 2018; 31: 135-137.

20. Grasel R, Santos MJ, Rêgo HC, Rippe MP, Valandro LF. Effect of resin luting systems and alumina particle air abrasion on bond strength to zirconia. Oper Dent 2018; 43: 282-290.

21. Liu D, Tsoi JK, Matinlinna JP, Wong HM. Effects of some chemical surface modifications on resin zirconia adhesion. J Mech Behav Biomed Mater 2015; 46: 23-30.
22. Casucci A, Monticelli F, Goracci C, Mazzitelli C, Cantoro A, Papacchini F, et al. Effect of surface pre-treatments on the zirconia ceramic-resin cement microtensile bond strength. Dent Mater 2011; 27: 1024-1030.

23. Keshvad A, Hakimaneh SM. Microtensile bond strength of a resin cement to silica-based and Y-TZP ceramics using different surface treatments. J Prosthodont 2018; 27: 67-74.

24. Chen C, Niu LN, Xie H, Zhang ZY, Zhou LQ, Jiao K, Chen JH, Pashley DH, Tay FR. Bonding of universal adhesives to dentine-Old wine in new bottles?. J Dent 2015; 43: 525-536.

25. Özcan M, Bernasconi M. Adhesion to zirconia used for dental restorations: a systematic review and meta-analysis. J Adhes Dent 2015; 17: 7-26.

26. Melo R, Souza R, Dursun E, Monteiro E, Valandro L, Bottino M. Surface treatments of zirconia to enhance bonding durability. Oper Dent 2015; 40: 636-643.

27. Lundberg K, Wu L, Papia E. The effect of grinding and/or Air-borne particle abrasion on the bond strength between zirconia and veneering porcelain: a systematic review. Acta Biomater Odontol Scand 2017; 28: 8-20.

28. Wang H, Aboushelib MN, Feilzer AJ. Strength influencing variables on CAD/CAM zirconia frameworks. Dent Mater 2008; 24: 633-638.

29. Sakrana AA, Özcan M. Effect of chemical etching solutions versus air abrasion on the adhesion of self-adhesive resin cement to IPS e. max ZirCAD with and without aging. Int J Esthet Dent 2017; 12: 72-85.

30. Zandparsa R, Talua NA, Finkelman MD, Schaus SE. An in vitro comparison of shear bond strength of zirconia to enamel using different surface treatments. J Prosthodont 2014; 23: 117-123.

31. Özcan M, Nijhuis H, Valandro LF. Effect of various surface conditioning methods on the adhesion of dual-cure resin cement with MDP functional monomer to zirconia after thermal aging. Dent Mater J 2008; 27: 99-104.

32. Yenisey M, Dede DÖ, Rona N. Effect of surface treatments on the bond strength between resin cement and differently sintered zirconium-oxide ceramics. J Prosthodont Res 2016; 60: 36-46.

33. Oguri T, Tamaki Y, Hotta Y, Miyazaki T. Effects of a convenient silica-coating treatment on shear bond strengths of porcelain veneers on zirconia-based ceramics. Dent Mater 2012; 31: 788-796. 
34. Samran A, Al-Ammari A, El Bahra S, Halboub E, Wille $\mathrm{S}$, Kern M. Bond strength durability of self-adhesive resin cements to zirconia ceramic: An in vitro study. J Prosthet Dent 2018.

35. Tanış MÇ,Akçaboy C. Effects of different surface treatment methods and MDP monomer on resin cementation of zirconia ceramics an in vitro study. Lasers Med Sci 2015; 6: 174-181.

36. Aboushelib MN, Kleverlaan CJ, Feilzer AJ. Selective infiltration-etching technique for a strong and durable bond of resin cements to zirconia-based materials. J Prosthet Dent 2007; 98: 379-388.

37. Akay C, Çakırbay Tanış M, Şen M. Effects of hot chemical etching and 10-metacryloxydecyl dihydrogen phosphate
(MDP) monomer on the bond strength of zirconia ceramics to resin-based cements. J Prosthodont. 2017; 26: 419-423.

38. Lüthy H,Loeffel O, Hammerle CH.Effect of thermocycling on bond strength of luting cements to zirconia ceramic. Dent Mater 2006; 22: 195-200.

39. Furuichi T, Takamizawa T, Tsujimoto A, Miyazaki M, Barkmeier W, Latta M. Mechanical properties and slidingimpact wear resistance of self-adhesive resin cements. Oper Dent 2016; 41: E83-E92.

40. Higashi M, Matsumoto M, Kawaguchi A, Miura J, Minamino T, Kabetani T, et al. Bonding effectiveness of selfadhesive and conventional-type adhesive resin cements to CAD/CAM resin blocks. Part 1: Effects of sandblasting and silanization. Dent Mater J 2016; 35: 21-28. 